\title{
Evaluation of Pattern of Angiogenesis in Various Menstrual Disorders in Different District in Gujarat, India
}

\author{
Killol Nathubhai Desai ${ }^{1 *}$ and Vidya Satapara ${ }^{2}$ \\ ${ }^{1}$ Department of Pathology, GMERS Medical College, Junagadh, Gujarat, India \\ ${ }^{2}$ Department of Anatomy, GMERS Medical College, Junagadh, Gujarat, India
}

\section{ABSTRACT}

Backgound: Menstrual disturbance is one of the commonest gynecological problems. Majority of these women have dysfunctional uterine bleeding (DUB), the rest being associated with leiomyomas, carcinomas, adenomyosis, endometrial polyps, hyperplasia and oral contraceptive uses.

Aims: To study of alteration in blood vessels morphology in various menstrual disorders have significant role in prognosis and in various anti-angiogenic therapies.

Methods and Material: A Cross-sectional study done at Anand, Surendranagar and Junagadh district from October 2011 to October 2016. 1867 endometrial specimens were studied to document the changes in blood vessels in various phases of menstrual cycle, menstrual disturbances and in unexplained infertility. 761 cases were taken as control and 1106 cases as study group which included cases of dysfunctional uterine bleeding (DUB), endometrial polyps, fibroids, adenomyosis, infertility, atrophic endometrium, endometrial hyperplasia, pill endometrium and carcinomas. Using light microscopy, the vascular morphology was studied. Statistical analyses were performed using the IBM SPSS (Statistical package for the Social Sciences v15.0) and Microsoft Excel 2007 software.

Results: The blood vessels were concentrated more in basal layer in the proliferative phase and in functional layer in the secretory phase. Cases of complex hyperplasia and pill endometrium had significantly higher vessel concentration. Congestion and dilatation of blood vessels were significantly higher in cases of DUB.

Conclusions: The present study showed a positive correlation between endometrial angiogenesis and menstrual disorders. The alteration in blood vessel morphology has significant role in prognosis and in various anti-angiogenic therapies.

Keywords: Angiogenesis, Menstrual Disorders, Dysfunctional Uterine Bleeding, Endometrium

\section{Introduction}

Menstrual disturbance is one of the commonest gynecological problems for which a curettage or hysterectomy specimen is received by the pathologists. Majority of these women have dysfunctional uterine bleeding (DUB) of unknown pathology, the rest being associated with leiomyomas, carcinomas, adenomyosis, endometrial polyps, hyperplasia and oral contraceptive uses. ${ }^{[1]}$ Poor understanding of the mechanisms underlying spontaneous and induced menstrual disturbances has hindered the development of non-surgical therapies for these complaints. Another problem encountered is that of unexplained infertility. Although some ultrastructural studies have been carried out in the past, they cannot form a part of routine endometrial evaluation in the infertility and other gynecological disorders. Therefore, the present study employed the routinely used stains to evaluate the number and morphology of endometrial blood vessels associated with benign gynecological conditions resulting from menstrual disturbances and infertility.

\section{Material and Methods}

This study was carried out in three different Medical Colleges from October 2011 to October 2016 like 1) Department of Pathology, Pramukhswami Medical College, Karamsad, Anand, Gujarat, India, 2) Department of Pathology, C.U. Shah Medical College, Surendranagar, Gujarat, India and 3) Department of Pathology, GMERS Medical College, Junagadh, Gujarat, India. (Table 1) A total of 1867 endometrial specimens obtained by hysterectomy and dilatation and curettage (D and C) were included in the study. 761 cases were taken as control and 1106 cases as study group ( Control taken from hysterectomy specimens which are operated for non-hormonal conditions, due to lesson of hysterectomy specimen for control in my time duration and further no necessity of same case and control group). In the control group, endometrial specimens were 
obtained from the hysterectomy specimens performed for non-hormonal indications like prolapse, endometrial polyps, fibroids and adenomyosis in young patients in the age group of 28-40 years who had a normal and regular menstrual cycle and from endometrial curettage performed along with cervical biopsies with no suspicion of hormonal endometrial pathology in young patients in the age group of 28-40 years. We are only used specimens which are received in our histopathology laboratory for diagnosis purpose and HREC of Pramukhswami Medical College, Karamsad, Anand, Gujarat, India give permission with waiver of consent. So consent of patient is not required in my study. The study group included cases of DUB and local uterine lesions like, endometrial polyps, fibroids and adenomyosis. Cases of infertility and atrophic endometrium were also studied. The pertinent patient details like age, hospital, menstrual history, obstetric history, chief complaints, history of hormonal use and the clinical diagnosis of the patient were also studied. Required sections were taken from the hysterectomy specimens. Specimens of D and C and suction-evacuation were examined for adequacy and those containing only blood were not included in the study. The tissues were fixed in $10 \%$ formalin and processed routinely. Sections of 3 micron thickness were stained with Hematoxylin and Eosin ( $\mathrm{H}$ and $\mathrm{E}$ ) stain. Wherever required van Gieson stain was done. The appropriate controls were used with each batch of van Gieson stain. Van Gieson stain used for staining of collagen and other connective tissue, so it is used in endometriosis, stromal endometrial fibrosis, periglandular and perivascular fibrosis. Sections stained by $\mathrm{H}$ and $\mathrm{E}$ stain were used for dating of the endometrium and evaluation of various characteristics of endometrial blood vessels with respect to number, congestion and degree of dilatation. The overall vascularity of the endometrium was judged by counting the average number of blood vessels seen in 10 high-power fields (HPFs) in basal layer and functional layer separately and compared with that of the controls. As the curettages contained endometrium of the functional layer, an average was calculated after observing $10 \mathrm{HPFs}$. The statistical test used for comparison was Student's unpaired $t$-test. $P$ value less than 0.05 was considered significant. Vascular congestion was noted for its presence or absence. The degree of dilatation was graded as mild and moderate. These values were compared with that of the controls. The statistical test used for comparison was $Z$ test. $P$ value less than 0.05 was considered significant.

\section{Results}

In 761 cases of the control group, average endometrial blood vessels per HPF in hysterectomy specimens are given in Table 3. There was statistically significant difference in endometrial blood vessel density in different phases of menstrual cycle in the basal and functional layers $(P<$ $0.05)$. The blood vessels are mainly concentrated in the basal layer in the proliferative phase and were concentrated more in the functional layer in the secretary phase [Figures 1 and 2]. However, there was no statistically significant difference between the average endometrial (basalis + functionalis) blood vessel concentration per HPF in various phases of menstrual cycle.

The study group included 1106 endometrial specimens of DUB, of which 369 were hysterectomy and 737 were D and $\mathrm{C}$ specimens. Some of the specimens also associated with atrophic uterus (14\%), leiomyoma (13\%), infertility $(4 \%)$, polyps (3\%) and adenomyosis $(3 \%)$.

In DUB, according to the histopathology diagnosis, the hysterectomy and $\mathrm{D}$ and $\mathrm{C}$ cases were distributed as in Table 2. The mean of the blood vessels per HPF in early proliferative, late proliferative, early secretory, mid secretory and late secretory were calculated together and placed into a group endometrium (proliferative + secretory) and compared with the control as in Table 4. Other histopathology categories included cystic glandular hyperplasia (CGH), simple hyperplasia, complex hyperplasia and pill endometrium. There was no statistically significant difference in blood vessel density in the hysterectomy specimens for DUB and control group. In $\mathrm{D}$ and $\mathrm{C}$ specimens, there was no significant difference between the study and control mean of average blood vessels per HPF in the group endometrium (proliferative + secretory), simple hyperplasia and CGH. The average blood vessels per HPF were significantly higher in complex hyperplasia (4.01 \pm 0.12$)$, pill endometrium $(6.42 \pm 0.67)$ and endometrial carcinoma ( $4.37 \pm 0.093)$ as compared to control (3.80 \pm 0.42$), P<0.01$, Table 4).

The number of cases in the group endometrium (proliferative + secretory) showed congestion was $43.58 \%$ as compared to $11.2 \%$ cases in the control group which was significantly higher $(P<0.001)$. Cases of CGH and simple hyperplasia that showed congestion were significantly higher $(55.3 \%)$, when compared to $11.2 \%$ control cases $(P<0.001)$. Seventy-five percent $(75 \%)$ of cases of complex hyperplasia showed significant congestion $(P<$ $0.001)$. Cases of pill endometrium also showed significant congestion $(P<0.001)$.

The number of cases showing mild dilatation in the group endometrium (proliferative + secretory) was $23.4 \%$ and moderate dilatation was $6.4 \%$. Both these figures were significantly higher as compared to control $(P<0.001)$. The number of CGH and simple hyperplasia cases showing mild dilatation was $30.5 \%$ and moderate dilatation was $9.5 \%$. These values were also significantly higher as compared to 
control [Figure 4]. In complex hyperplasia, mild dilatation and moderate dilatation were significant as compared to control. The percentage of pill endometrium cases showing mild blood vessel dilatation was $57.5 \%$ which was also significantly higher than in control [Figure 3].

In cases of fibroids (13\%), the mean of the blood vessels in the stratum basalis and stratum functionalis was not significantly different as compared to the controls. The endometrial blood vessel congestion was $13.2 \%$ as compared to control (12.7\%) which was not significantly different $(P>0.05)$. No significant difference in dilatation was seen between the two groups.

In the cases of adenomyosis (3\%) were included in the study. All were hysterectomy specimens. No significant difference was observed in the mean of blood vessel concentration in adenomyosis as compared to control.
In the cases of endometrial polyp (3\%) were studied. The mean blood vessel concentration in polyp was $(5.12 \pm 0.77)$ which was significantly higher $(P<0.05)$ as compared to control. The stalk of the endometrial polyp showed the presence of large, thick-walled muscular arteries with wellcollagenized adventitial coat brightly staining red by van Gieson stain. Prominent arterioles were also seen beneath the surface lining epithelium.

In the cases of infertility (4\%) were included in the study. All were D and C specimens. There was no significant difference between the mean blood vessel concentration of control and infertility cases. None of the cases showed blood vessel congestion or dilatation.

In the cases of atrophic endometrium (14\%) were studied. The mean of average blood vessel concentration was not significantly different when compared to control.

Table 1: District wise case and control

\begin{tabular}{|l|c|c|c|}
\hline District & Case & Control & Total \\
\hline Anand & 533 & 303 & 836 \\
\hline Surendranagar & 206 & 201 & 407 \\
\hline Junagadh & 367 & 257 & 324 \\
\hline Total & $\mathbf{1 1 0 6}$ & $\mathbf{7 6 1}$ & $\mathbf{1 8 6 7}$ \\
\hline
\end{tabular}

Table 2: Histopathology diagnosis-wise distribution of dysfunctional uterine bleeding group (Case)

\begin{tabular}{|c|c|c|c|}
\hline Histopathology diagnosis & Hysterectomy (n) & $\mathrm{D} \& \mathrm{C}(\mathrm{n})$ & Total \\
\hline Early proliferative & 52 & 126 & 178 \\
\hline Late proliferative & 30 & 126 & 156 \\
\hline Early secretory & 40 & 94 & 134 \\
\hline Mid secretory & 33 & 113 & 146 \\
\hline Late secretory & 20 & 58 & 78 \\
\hline Cystic glandular hyperplasia & 12 & 84 & 96 \\
\hline Simple endometrial hyperplasia (Typical+Atypical) & 97 & 97 & 194 \\
\hline Complex endometrial hyperplasia (Typical+Atypical) & 38 & 06 & 44 \\
\hline Pill endometrium & 26 & 30 & 56 \\
\hline Endometrial carcinoma & 21 & 03 & 24 \\
\hline Total & 369 & 737 & 1106 \\
\hline
\end{tabular}

Table 3: Average endometrial blood vessels in control group

\begin{tabular}{|l|c|c|c|}
\hline \multirow{2}{*}{ Histopathology diagnosis } & \multicolumn{2}{|c|}{ Average blood vessels/HPF* (Mean) } & $\begin{array}{c}\text { Basalis + Functionalis } \\
\text { (Mean) }\end{array}$ \\
\cline { 2 - 4 } & Basalis & Functionalis & $3.23 \pm 0.3231$ \\
\hline Early proliferative & $3.85 \pm 0.2261$ & $2.60 \pm 0.4200$ & $3.65 \pm 0.3274$ \\
\hline Late proliferative & $3.62 \pm 0.3881$ & $3.68 \pm 0.2666$ & $3.64 \pm 0.3098$ \\
\hline Mid secretory & $3.28 \pm 0.2865$ & $4.0 \pm 0.3331$ & $3.41 \pm 0.5622$ \\
\hline Late secretory & $3.21 \pm 0.4412$ & $3.60 \pm 0.6832$ & $3.16 \pm 0.6106$ \\
\hline Total Mean & $2.62 \pm 0.5212$ & $3.70 \pm 0.7000$ & $\mathbf{3 . 4 1 \pm 0 . 4 2 6 6}$ \\
\hline
\end{tabular}

*HPF- High Power Field 
Table 4: Comparison of average endometrial blood vessels per high power field in case of Dysfunctional uterine bleeding.

\begin{tabular}{|c|c|c|c|}
\hline \multirow{2}{*}{ Histopathology diagnosis } & \multicolumn{2}{|c|}{ Mean } & \multirow{2}{*}{ p-Value } \\
\hline & DUB* $^{*}$ & Control & \\
\hline Proliferative + secretory endometrium & $3.84 \pm 0.34$ & $3.80 \pm 0.42$ & $>0.05 \mathrm{NS}^{* \star *}$ \\
\hline Simple endometrial hyperplasia (Typical+Atypical) & $3.92 \pm 0.42$ & $3.80 \pm 0.42$ & $>0.05 \mathrm{NS}^{* * *}$ \\
\hline Cystic glandular hyperplasia & $3.90 \pm 0.22$ & $3.80 \pm 0.42$ & $>0.05 \mathrm{NS}^{* * *}$ \\
\hline $\begin{array}{l}\text { Complex endometrial hyperplasia } \\
\text { (Typical+Atypical) }\end{array}$ & $4.01 \pm 0.12$ & $3.80 \pm 0.42$ & $<0.01 \mathrm{HS}^{* * *}$ \\
\hline Pill endometrium & $6.42 \pm 0.67$ & $3.80 \pm 0.42$ & $<0.001 \mathrm{VHS}^{* * *}$ \\
\hline Endometrial carcinoma & $4.37 \pm 0.09$ & $3.80 \pm 0.42$ & $<0.01 \mathrm{HS}^{* * *}$ \\
\hline
\end{tabular}

${ }^{*} D U B$ - Dysfunctional uterine bleeding, **CGH - Cystic glandular hyperplasia,

*** NS - Not significance, $H S=H i g h$ significance, $V H S=$ Very high significance

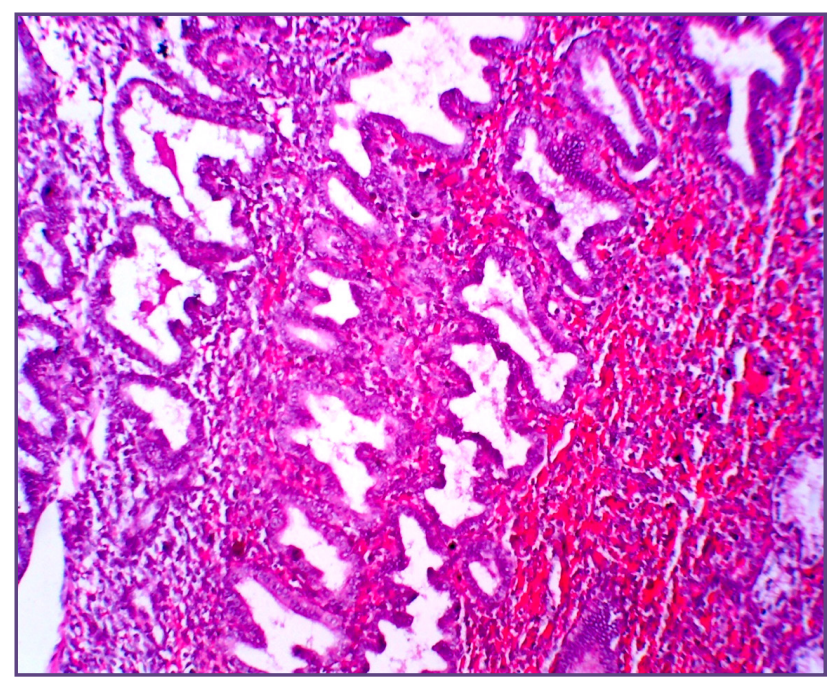

Fig. 1: Microphotograph of Secretory endometrium (H\&E stain $10 x$ view).

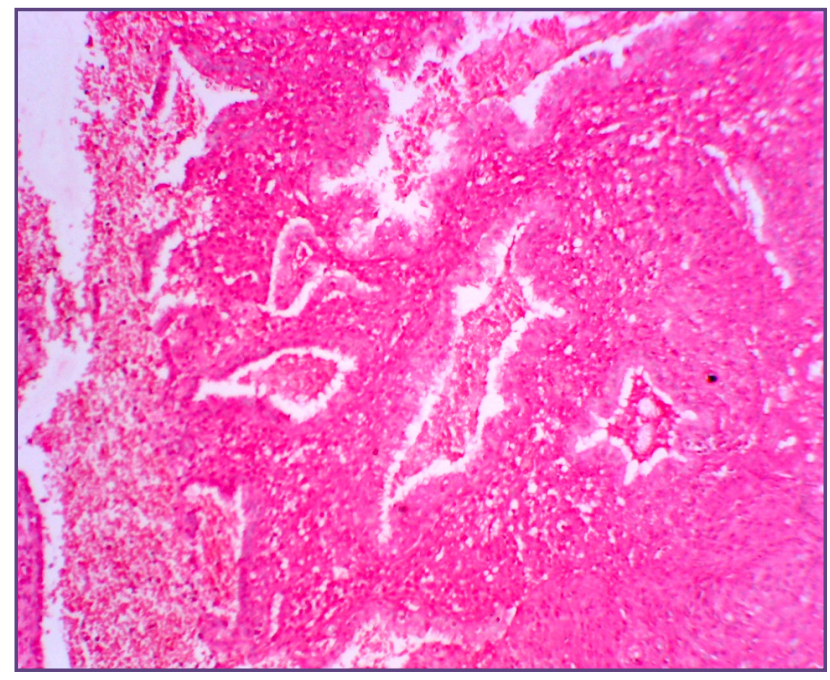

Fig. 3: Microphotograph of Hormonal induced changes of endometrium (Pill endometrium) (H\&E stain 10x view)

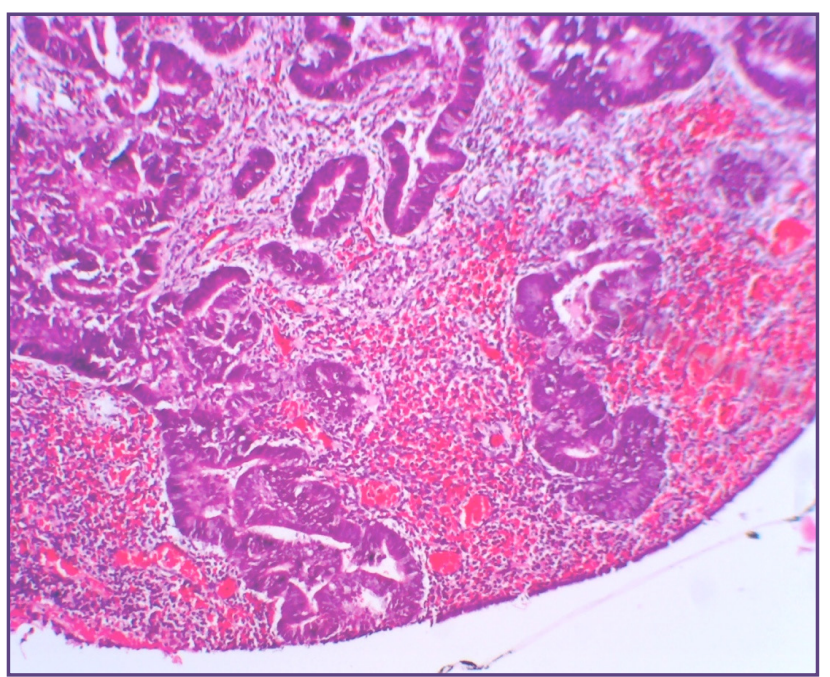

Fig. 2: Microphotograph of Proliferative endometrium (H\&E stain 10x view).

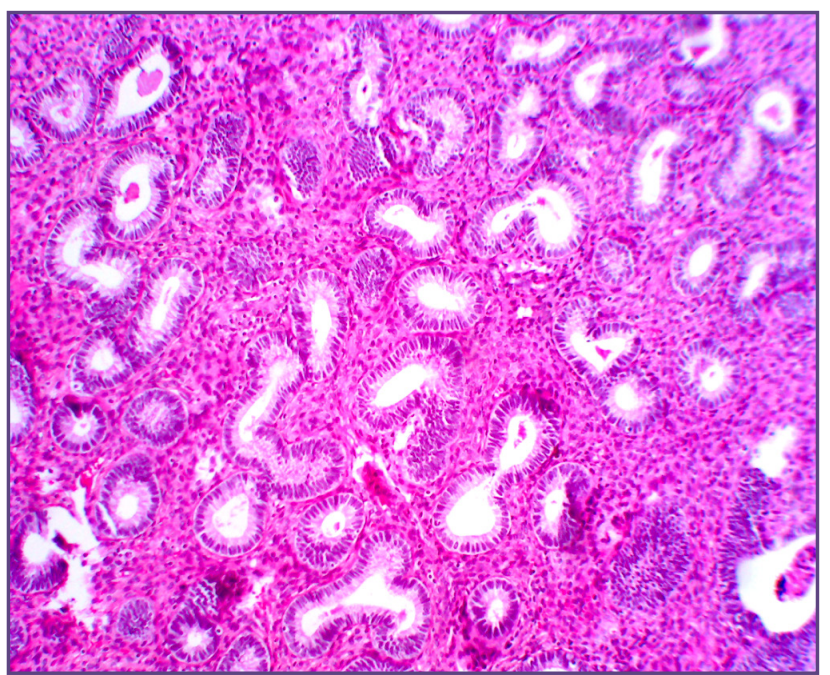

Fig 4: Microphotograph of Simple glandular hyperplasia of endometrium (H\&E stain 10x view). 


\section{Discussion}

Endometrial angiogenesis is characterized by proliferation of vascular endothelial cells during proliferative phase, coiling of arterial system in secretory phase and repair of vascular bed during menstruation. Excessive or insufficient vascular growth contributes to numerous benign disorders. In many angiogenesis related diseases, there is defective remodeling of vascular bed and changes in vascular fragility. ${ }^{[2]}$

In the present study, the endometrial blood vessels were evaluated using light microscopy on sections stained by $\mathrm{H}$ and $\mathrm{E}$ stain. This method was chosen because in the routine set-up, it provides maximum information and utility and can be readily applied in the study of endometrial vasculature as observed by Upadhyay and Mishra $^{[3]}$ and Sahasrabudhe et al..$^{[4]}$

The method used for the counting of blood vessels was obtained from Hourihan et al. ${ }^{[5]}$ and Sahasrabudhe et $a l .{ }^{[4]}$ The endometrium in the control group was dated as early proliferative, late proliferative, early secretory, mid secretory and late secretory. The average number of blood vessels per HPF for hysterectomy cases was $3.54 \pm 0.20$, which was comparable to that observed by Sahasrabudhe et $a l .{ }^{[4]}$ but lower as compared to the study of Shaw et al ${ }^{[6]}$ The blood vessels were concentrated more in the basal layer in proliferative endometrium and were distributed more in the functional layer by mid to late secretory phase, which was in agreement with those of Fanger and Barker, ${ }^{[7]}$ Sahasrabudhe et al., ${ }^{[4]}$ and Nayha et al. ${ }^{[8]}$ This is because, the spiral arterioles are extremely sensitive to the changes in levels of ovarian hormones and changes occur in the vessel density as the cycle progresses. However, there was no significant change in the average density of blood vessels (basalis + functionalis) during the various phases of the menstrual cycle like in the study done by Shaw et al. ${ }^{[6]}$ and Rees et al. ${ }^{[9]}$ This is because other elements such as glandular and stromal cells also increase in size and number in proportion to vascular growth in maturing endometrium and the relative number of blood vessels remains constant. ${ }^{[6]}$

In cases of DUB, the mean blood vessels per HPF in complex hyperplasia were significantly higher as compared to control $(P<0.001)$ as observed by Abulafia et al.$^{[10]}$ This is due to significant increase in endothelial cell proliferation in the endometria of women with menorrhagia. ${ }^{[1]}$ Similar finding was noted by Nayha et al. and they indicated that this microvascular density is associated with malignant transformation later. ${ }^{[8]}$ The difference in the blood vessel mean $(6.21 \pm 0.27)$ of pill endometrium as compared to control (3.86 \pm 0.36$)$ was significantly higher as claimed by
Hourihan et al..$^{[5]}$ and Hickey et al. ${ }^{[12]}$ However, the blood vessels were thin walled and congested. This effect reflects a combined estrogen progesterone effect which typically produces a weakly secretory pattern characterized by under developed non-coiled glands set within a spindled, vaguely predeciduated stroma containing thin-walled vascular channels. The increased density of blood vessels combined with the fragile nature of these vessels may be responsible for the hormone-induced breakthrough bleeding. Therefore, except for pill endometrium and complex hyperplasia, no significant difference was found in the concentration of blood vessels in curettage and hysterectomy specimens of DUB cases as compared to control which was in agreement with those of Rees et al., ${ }^{[9]}$ Hourihan et al., ${ }^{[5]}$ Sahasrabudhe et al., ${ }^{[4]}$ and Mints et al. ${ }^{[2]}$ Therefore, it is possible that excessive bleeding in DUB may be related to other qualitative changes in the blood vessels.

Congestion was significantly higher in cases of DUB in the group endometrium (proliferative + secretory), $\mathrm{CGH}$, complex hyperplasia and pill endometrium when compared with the controls .

Vascular dilatation was graded as mild and moderate. Mild dilatation was significantly increased in cases of group endometrium, $\mathrm{CGH}$, complex hyperplasia and pill endometrium. Moderate dilatation was also increased in cases of group endometrium and $\mathrm{CGH}$.

Sippe noted the presence of dilated vascular sinuses in $77 \%$ of cases of endometrial hyperplasia. ${ }^{[13]}$ In the present study, such sinuses were found in $59.53 \%$ of cases including CGH and complex hyperplasia and $25.8 \%$ of cases of group endometrium (proliferative + secretory). This lower incidence is because unless the curettage or hysterectomy is carried out during an actual bleeding episode, a certain number of cases will not show this vascular changes. ${ }^{[13]}$ However, similar lower incidence of dilated vascular channels $(25 \%)$ was observed by Sahasrabudhe et al. ${ }^{[4]}$ Thus, it is possible that the various morphological changes in the endometrial vasculature in DUB and rupture of the dilated and congested vascular channels could be responsible for the abnormal uterine bleeding.

In cases of fibroids, there was no significant difference in the mean of the blood vessels, congestion or dilatation when compared with the controls as seen in the study carried out by Sahasrabudhe et al. ${ }^{[4]}$ This is in contrast to the findings of Farrer-Brown et al., where dilated channels were noted in all cases of leiomyomatous uteri, ${ }^{[14]}$ which was an injection study that might have distended the normally collapsed vascular channels by the injection material. ${ }^{[4]}$ 
The cases of adenomyosis showed reduced vascularity in the adenomyotic foci as compared to the controls which was in agreement with that of Beilby et al. ${ }^{[15]}$ This is contradictory due to the fact that adenomyosis is associated with abnormal uterine hemorrhage in a significant number of cases.

In cases of endometrial polyp, the average blood vessel concentration was significantly higher as compared to control. This was in agreement with that of Beilby et al. ${ }^{[15]}$ and Sahasrabudhe et al..$^{[4]}$ The large thick-walled muscular arteries with well-collagenized adventitial coat highlighted by van Gieson stain, which are never seen normally in the endometrium, were present mainly in the stalk of the polyp. Prominent arterioles were seen even beneath the surface lining epithelium. These morphological features find diagnostic application especially when a fragmented polyp is curetted.

In the cases of infertility showed no statistically significant difference in the mean of blood vessels, congestion, or dilatation when compared with the control group. Thus, the present study could not establish inadequate vascularization of the endometrium as one of the factors involved in unexplained infertility as observed by Sahasrabudhe et $a l .{ }^{[4]}$ Deficient blood flow to the endometrium as studied by Edi-Osagie et al. and Steer et al., might be the reason in such cases. ${ }^{[16,17]}$

In atrophic endometrium, mean of average blood vessel concentration was slightly lower as compared to control, but not statistically significant that was also observed by Hickey et al. ${ }^{[18]}$ The endometrial glands were lined by mitotically inactive bland epithelium in a similar spindled inactive stroma. In some cases, the glands were cystically dilated and lined by cuboidal to flattened epithelium. These effects are due to withdrawal of endogenous ovarian steroids on the endometrial blood vessels.

\section{Conclusion}

The endometrial blood vessels showed characteristic changes in various phases of menstrual cycle. They were concentrated more in basal layer in the proliferative phase and were distributed more in functional layer in the secretory phase. The average endometrial blood vessels/ HPF in complex hyperplasia and pill endometrium were significantly higher when compared with the controls. The endometrial blood vessel congestion was significantly high in the group endometrium (proliferative + secretory), complex hyperplasia and pill endometrium when compared to the controls. The endometrial blood vessel dilatation was significantly high in the group endometrium (proliferative + secretory), complex hyperplasia and pill endometrium when compared with the controls.
The present study showed a positive correlation between endometrial angiogenesis and menstrual disorders. In the present era of newer anti-angiogenic therapies, endometrial angiogenesis and alteration in vascular morphology definitely has prognostic significance and thus helps to improve the treatment modalities and patient care. Early diagnosis of menstrual disorders improve patient's symptoms, signs and newer anti-angiogenic therapies very effective at early stage of menstrual disorders. They also decrease probability of operative treatment procedure.

\section{References}

1. Sherman ME, Mazur MT, Kurman RJ. Benign diseases of the endometrium. In: Kurman RJ. Blaustein's pathology of the female genital tract, 5th ed. USA: Springer; 2002. 421-66.

2. Mints M, Blomgren B, Falconer C, Fianu-Jonasson A, Plamblad J.Microvascular density, vascular endothelial growth factor A and its receptors in endometrial blood vessels in patients with menorrhagia. Fertil Steril 2005;84:692-700.

3. Upadhyay SN, Mishra J. Observations on histopathological changes in the uterus in dysfunctional uterine bleeding. J Obstet Gynaecol India 1963;13:531-41.

4. Sahasrabudhe NS, Dalal SR, Jadhav MV. Endometrial blood vessels in health and disease. Med J West India 2000;28:27-31.

5. Hourihan HM, Sheppard BL, Bonnar J. A morphometric study of the effect of oral norethisterone or levonoregestrel on endometrial blood vessels. Contraception 1986;34:603-12.

6. Shaw ST, Macaulay LK, Hohman WR. Vessel density in endometrium of women with and without intrauterine contraceptive devices: a morphometric evaluation. Am J Obstet Gynecol 1979;135:202-6.

7. Fanger H, Barker BE. Capillaries and arterioles in normal endometrium. Obstet Gynaecol 1961;17:543-50.

8. Nayha V, Viitanen T, Stenback F. Altered extent, pattern and characteristics of microvascular density are indicators of Neoplastic progression in the endometrium. Int J Cancer 2005;115:975-80.

9. Rees MC, Dunhill MS anderson AB, Turnbull AC. Quantitative uterine histology during the menstrual cycle in relation to measured menstrual blood loss. Br J Obstet Gynaecol 1984;91:662-6.

10. Abulafia O, Triest WE, Sherer DM, Hansen CC, Ghezzi F. Angiogenesis in endometrial hyperplasia and stage I endometrial carcinoma. Obstet Gynecol 1995;86:479-85.

11. Abulafia O, Sherer DM. Angiogenesis of the endometrium. 1999;94:148-53.

12. Hickey M, Fraser IS. Clinical implications of disturbances of uterine vascular morphology and function. Baillière's Clin Obstet Gynecol 2000;14:937-51. 
13. Sippe G. Endometrial hyperplasia and uterine bleeding. J Obstet Gynaecol 1962;69:1015-9.

14. Farrer-Brown G, Beilby JOW, Tarbit MH. The vascular patterns in myomatous uteri. J Obstet Gynaecol Br Commonw 1970;77:967-75.

15. Beilby JO, Farrer-Brown G, Tarbit MH. The microvasculature of common uterine abnormalities, other than fibroids. J Obstet Gynaecol Br Commonw 1971;78:361-8.

16. Edi-Osagie EC, Seif MW, Aplin JD, Jones CJ, Wilson $\mathrm{G}$, Lieberman BA. Characterizing the endometrium in unexplained and tubal factor infertility: a multiparametric investigation. Fertil Steril 2004:82:1379-89.

17. Steer CV, Tan SL, Mason BA, Campbell S. Midluteal-phase vaginal color doppler assessment of uterine artery impedance in a subfertile population. Fertil Steril 1994;61:53-8.

18. Hickey M, Lau TM, Russell P, Fraser IS, Rogers PA. Microvascular density in conditions of endometrial atrophy. Hum Reprod 1996;11:2009-13.

*Corresponding author:

Dr. Killol Nathubhai Desai, A-301, Staff Quarters, GMERS Medical College, Paddock road, Junagadh, Gujarat, India. Pin: 362001,

Phone: +91 9428050253

Email: drkilloldesai@gmail.com

Date of Submission : 23.11.2016

Date of Acceptance : 12.02.2017

Financial or other Competing Interests: None.

Date of Publication : 05.06.2017 